\title{
Microscopy for Detection of Extant Life
}

A Science White Paper for the Planetary Science Decadal Survey

\author{
Topic Areas: \\ Life and Prebiotic Organics \\ Ocean Worlds \\ Technology Development
}
Authors and Affiliations:
Christian Lindensmith Jet Propulsion Laboratory, California Institute of Technology
Christian.A.Lindensmith@jpl.nasa.gov
Jay Nadeau, Portland State University
nadeau@pdx.edu
Eugene Serabyn, Jet Propulsion Laboratory, California Institute of Technology
eugene.serabyn@.jpl.nasa.gov
Peter Willis, Jet Propulsion Laboratory, California Institute of Technology
peter.a.willis@jpl.nasa.gov
Penelope Boston, NASA Ames Research Center
penelope.j.boston@nasa.gov
Michael Spilde, University of New Mexico
mspilde@unm.edu

\section{Primary Author Contact}

Christian Lindensmith Jet Propulsion Laboratory, California Institute of Technology

Christian.A.Lindensmith@,jpl.nasa.gov

(818) 354-6697

The work described in this paper was done in part at the Jet Propulsion Laboratory, California Institute of Technology, under contract with the National Aeronautics and Space Administration. (C) 2020. All rights reserved. 
The central question of astrobiology, "Is there life elsewhere in the universe?" in the context of this decadal survey transforms to "Is life present on any other planet or moon in our own solar system?" The last time that NASA sent an in situ mission with instruments designed to search for extant life was the Viking mission, in which the "Labeled Release" experiments returned results that were ambiguous at best, and likely to have been the result of abiotic processes ${ }^{1,2}$. Work by numerous groups and individuals since Viking has led to current models of the search for life based on multiple independent lines of evidence ${ }^{3-5}$. There remains no well-defined, agreed-upon set of measurements that will provide a simple yes/no answer as to the presence of extant microbial life. Because we can't know in advance what extraterrestrial life might look like, or what specific chemistry it might use, multiple lines of evidence on a shared sample are essential to provide a much higher level of confidence in a claim for the detection of extant life than is possible from any single instrument. Life is a highly complex phenomenon, and thus, not amenable to a single "smoking gun" marker that can provide an unequivocal indication of life processes. Rather, suites of instruments and data types are required to provide adequate basis for making claims of life detection. As described in the "The Ladder of Life Detection", many indicators may provide strong evidence, but no single indicator is expected to be detectable in all cases, even with Earth life.

We recommend that NASA and the astrobiology community pursue the development of integrated suites of instruments, or a framework of independent instrument development for integration into a suite, that includes multiple chemical and microscopic analysis approaches. Because the science and technology of such an integrated approach is broader in scope than can fit into a single white paper we divide the discussion into two complementary white papers, one for chemical analyses (see white paper by Willis, et al.) and the present one describing the science value and the technology status and needs toward the development of microscopic imaging for extant life detection.

\section{Role of Microscopy in Microbiology}

Microscopy is at the center of the discovery and investigation of microbial life on earth. From the earliest observations by Leeuwenhoek and Hooke through modern applications in molecular biology $^{6}$ and super-resolution molecular imaging ${ }^{7-9}$, microscopy has provided stunning images of the microbial world, leading to breakthroughs in our understanding. To state the obvious, microscopic observations have the great advantage of being able to directly see whole cells alive, intact, and functioning. The current state of affairs is vastly different from that described by Lovelock in his seminal paper on life detection, where he pointed out that although " $a$ visual search...is nevertheless the most rapid and effective method of life recognition in terms of orderliness outside the bounds of random assembly," "with 1965 technology it was likely too complex for use in potential space missions and instruments as conceived at the time. Since then, only a limited number of micro-imagers have been flown on space missions: a number of optical systems with insufficient resolution to detect terrestrial bacteria, and two atomic force microscopes with extremely high resolution and essentially no survey capability. The Europa Lander study team recognized the need for development of microscopes for life detection as a key finding in their report ${ }^{4}$. The "ladder of life" framework ${ }^{3}$ also identifies microscopy as a key capability in identifying extant life, in contrast to detection markers that indicate biotic origin without direct evidence of extant life.

The development of modern CCD and CMOS detectors, compact solid-state lasers and LEDs, and fast computing with large data storage, has allowed microscopic imaging to break free of its previous constraints. Volumetric imaging techniques can eliminate the need for the expert users previously required to prepare samples, locate features, and describe or photograph them. Further 
recent developments in advanced microscopy enable us to achieve high resolution with sufficient sample throughput to effectively survey very sparsely populated environments with a significant chance of detecting microbial life, if present ${ }^{11}$.

In the first part of this white paper, we survey the information that microscopy can provide that is relevant to detection of extant life. In the second part, we describe the types of imagers needed to obtain these data. In the third part, we identify technology needs and their current status and provide a notional roadmap for micro-imaging for life detection. Throughout this white paper we focus on science and technology capable of detecting the smallest cells that might be anticipated in an extreme environment elsewhere in our solar system, with dimensions in the $0.2 \mu \mathrm{m}$ to $5 \mu \mathrm{m}$ range, and with sensitivity to very low concentrations $(\sim 100$ cells $/ \mathrm{mL})$ that may be present in extremely low-energy and low-nutrient environments.

\section{Microscopic Observations to Search for Extant Life}

Microscopy affords straightforward imaging observation of a number of characteristics of a sample, beyond the fundamental ones of cellular morphology and internal structure. The number, size distribution, and geometries of any small particles observed are readily determined, at least to the limits of the system resolution. With optical microscopy, multi-wavelength observations can be used to identify pigmentation, and thus some chemical information, whether with simple RGB filters or custom tuned filters for particular pigments. When combined with intrinsic fluorescence, fluorescent dyes and appropriate illumination sources, such multi-wavelength systems can be used to detect the presence of many specific classes of molecule, even well below the resolution limit. Lipids are likely to be necessary for any sort of cellular biology to support the compartmentalization that isolates the homeostatic insides of cells from their external environments. Proteins perform many functions in Earth organisms, serving as the building blocks of various cellular structures including ion channels and flagella or cilia, and may well be present in some form on extra-terrestrial cells as well. Nucleic acids are present in all terrestrial cells as the information-carrying molecules, and some related molecules may be present in extraterrestrial cells. Dyes that bind to broad selections of molecules in each of these categories are available and can be used as indicators that microscopic objects bear complex chemicals associated with life.

Active motion (swimming, gliding, twitching) and passive motion (Brownian, floating, sinking), obtained from time series imaging in 3D, and index of refraction measurements can discriminate whether an object is mineral-like or vesicle-like. Most microbes in Earth's aqueous environments have densities very close to water, while virtually all minerals have much higher densities ${ }^{12}$. Index of refraction shows similar differentiation between microbial cells (index close to water) and minerals (much higher indices) ${ }^{13}$, and at a minimum would distinguish liquid-filled vesicles from minerals. Optical polarizing characteristics of cells can be used as a method of assessing asymmetries in the abundance of chiral molecules, adding to evidence to the interpretation of a biotic or abiotic origin of a sample.

Some of the highest resolution types of microscopy can also be used to determine mechanical and chemical properties of objects at small scales. Atomic force microscopy can discriminate vesicles from mineral grains ${ }^{14}$, as well as obtain detailed morphology, while electron microscopy can potentially measure both morphology and elemental composition.

\section{Types of microscopy \& characteristics}

Optical. Optical microscopy is the workhorse of Earth-based microbiology. First used to detect microbial life more than 400 years ago, various forms of optical microscopy remain standard tools for the identification and characterization of microorganisms. Building a microscope that can re- 
solve small eukaryotes such as diatoms and algae, $10 \mu \mathrm{m}$ or more in diameter, is straightforward even in field instruments. Prokaryotes, i.e. bacteria and archaea, are much more difficult to resolve as they lack intracellular features (organelles) and are near the limit of resolution of light microscopy. They are also largely transparent, so either phase contrast or colorimetric stains are used to visualize them. Stains can distinguish bacteria from surrounding substrates and tissues, which formed the basis for discovery of the causes of many infectious diseases. Bacterial cell walls respond to stains depending upon their composition, leading to key techniques such as Gram staining and acid-fast staining. These techniques have largely been supplanted by fluorescence microscopy in recent years, but may prove useful for astrobiological life detection.

Newer techniques in optical microscopy are capable of detecting transparent objects without dyes, eliminating the need to guess the possible chemistry of unknown life in an alien environment, with the corresponding loss of insight into their chemical composition. These techniques, such as Digital Holographic Microscopy (DHM) ${ }^{15}$ and Fourier Ptychographic Microscopy (FPM) ${ }^{16}$ do this by acquiring images in ways that allow reconstruction of the phase and amplitude of the light throughout the volume of interest. This provides the complete image data, including the necessary phase information to compute phase-contrast images of transparent objects. DHM also provides optical data compression as part of the recording process, while FPM records many images and reduces the amount of data as part of pre-processing. Time series data from volumetric optical microscopes can be used to evaluate motion that may indicate biological activity.

Fluorescence. Fluorescence microscopy usually uses dyes that target specific chemical groups or molecule types. For bacterial enumeration on Earth, the most common dyes target nucleic acids. The ubiquitous "Live/Dead" stain uses a combination of DNA-targeting dyes, one of which (the "Live") is cell-permeant. The "Dead" stain is impermeant and only stains cells with compromised membranes. Other commonly used nucleic acid dyes are 4',6-diamidino-2-phenylindole (DAPI) and acridine orange (AO); $\mathrm{AO}$ fluoresces red when bound to RNA and green when bound to DNA. A key advantage of these dyes is that their quantum yield is substantially enhanced when they bind to their targets, by factors of 10 to $>1000$. This means that washing away excess dye is rarely necessary, and the dyes may be applied to solid substrates such as rocks without creating background (Fig $1 \mathrm{~A}$ ). There is emerging interest in the possibility of label-free (i.e intrinsic) fluorescence for life detection. This is an area that should be pursued by astrobiologists. The primary reason to avoid the use of dyes on missions is planetary protection: dyes are complex organics
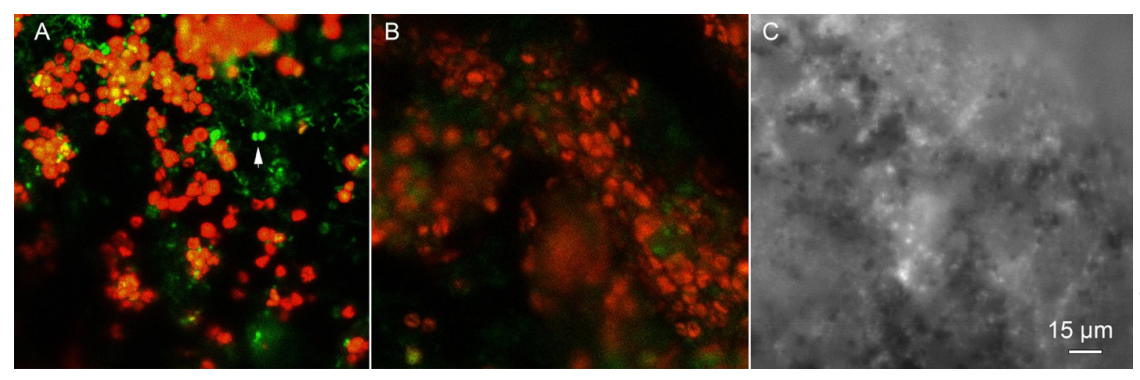

Figure 1. Desert sandstone Mars analog sample with and without dye. (A) Confocal image of rock containing photosynthetic cyanobacteria and unknown other organisms, labeled with SYTO9 (“Live”) (Ex: 488; Em 550$750 \mathrm{~nm})$.

Red shows cyanobacteria, and green shows living bacteria with DNA labeling. The arrow indicates non-photosynthetic cells. Smaller bacteria are visible above it. Many of the cyanobacteria appear yellow in the center due to the overlap of red and green channels. (B) Unlabeled area of the same sample, same imaging conditions as in (A). Cyanobacteria are still clearly seen, but the smaller organisms are invisible. (C) UV image of the same sample, excitation $280 \mathrm{~nm}$, emission 305-400 $\mathrm{nm}$. The cyanobacteria appear dark. Bright areas are unidentified and likely mineral in origin. 
that could look like biosignatures to chemical sensors. Other reasons include added complexity of fluidics required to stain samples and the Earth-centric nature of the dyes. Label-free fluorescence requires the ability to excite autofluorescent molecules within the cells, which have different properties depending upon the molecules present. Photosynthetic organisms are generally intensely autofluorescent due to the presence of complex rings including chlorophylls and other molecules. Chlorophylls a and b have a very strong absorbance peak in the blue and smaller peaks in the green to yellow region, with strong emission in the red (Fig. 1B). Phototrophic bacteria and algae are readily detected using label-free methods ${ }^{17}$, which form the basis of algae-counting technologies such as the flow cytobot ${ }^{18}$. Reduced nicotinamide adenine dinucleotide (NADH), with excitation/ emission $\sim 360 / 475 \mathrm{~nm}$, is widely used in cell biology as a fluorescent metabolic indicator and can be used to detect airborne bacteria. ${ }^{19}$ Aromatic amino acids (e.g., Typtophan (Trp), tyrosine (Tyr), phenylalanine (Phe)) and the proteins that contain them also fluoresce. Protein autofluorescence has attracted interest by astrobiologists but the signal is weak and requires ultraviolet (UV) excitation (excitation at $274-280 \mathrm{~nm}$ and emission at 303-348 nm). These signals are weak and nonspecific (Fig. 1C) and rarely used for bacterial detection; other molecules such as flavins show stronger signals and are attracting attention among microbiologists ${ }^{20,21}$.

UV imaging poses particular issues in liquids. Any medium containing organics has to be removed from the cells, because aromatic compounds within the medium can interfere with cell imaging (Fig 2). Minerals and mineral inclusions within rocks also show fluorescence emission peaks spanning the visible when excited in the UV; some of these signals arise from the minerals themselves and some from organics. Using information about a sample's fluorescent lifetime is another technique to better understand the origin of a fluorescence signal. Fluorescence lifeteime imaging microscopy (FLIM) and time-gated fluorescence imaging are two techniques that are commonly employed to this end. Label-free FLIM using NADH is a common technique in cell biology and provides information about cell metabolism. ${ }^{22}$

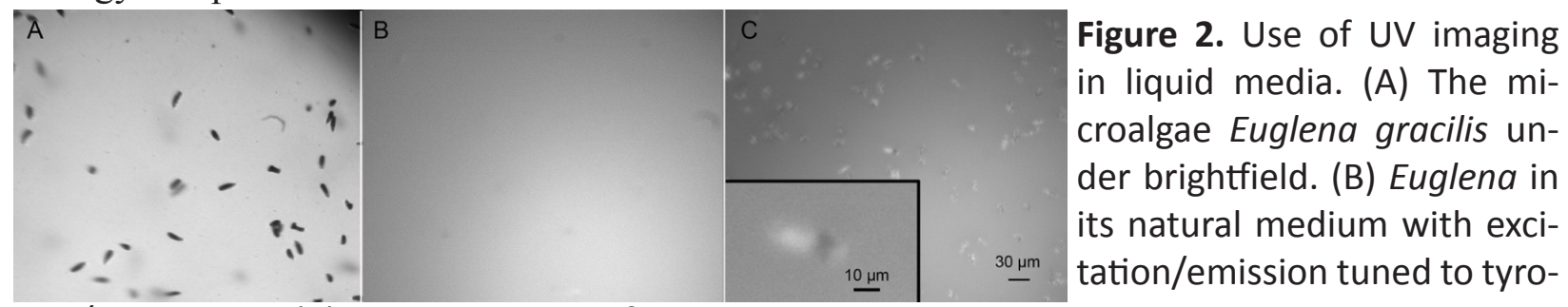

sine/tryptophan. (C) Euglena culture after 3 washes in distilled water. The zoom shows an individual cell; the dark area is chlorophyll.

Electron. Electron microscopy (EM) is widely used by microbiologists to examine microbial ultrastructure and identify the smallest cells, including so-called "nanobacteria" and viruses. Environmental scanning EM (ESEM) permits samples to be held under low-pressure water vapor with minimal sample preparation. A miniaturized ESEM was developed with variable-pressure capability for Mars exploration ${ }^{23}$, and the SEM and Particle Analyzer (SEMPA) included SEM and energy-dispersive $\mathrm{x}$-ray spectroscopy (EDS) and had $\sim 40 \mathrm{~m}$ spatial resolution with a $12 \mathrm{~kg} / 22 \mathrm{~W}$ payload $^{24}$. A bread-box sized instrument has been developed for the International Space Station and proposed for Mars ${ }^{25}$. SEM images of bacteria in microminerals can be ambiguous; EDS is often used simultaneously to provide elemental composition information about a sample, though it is sensitive to higher molecular weight elements so is more useful for identifying minerals than in disambiguating low-molecular weight material (Fig. 3A).

Transmission electron microscopy (TEM) can provide details of cell structure highly sugges- 
tive of life, such as flagella and pili (Fig. 3B). Stains are not necessary, though they do improve contrast. Scanning TEM (STEM) can disambiguate cells from minerals (Fig. 3C). Unlike SEM, TEM instruments have not been miniaturized. The difficulty in miniaturizing EM for space flight lies in the size of the electron gun and the power required to accelerate the electrons.

Sample throughput is the major drawback of electron microscopy - the imaged volume is so small that candidate objects need be identified by other means and brought into the field of the EM. Complex sample processing is also often necessary (embedding, staining, and thin-sectioning), except in the case shown of sparse organisms in liquid media. Viruses are particularly straightforward to image by TEM, and so there is significant interest by astrovirologists in developing instruments that could be used to identify viruses on Ocean Worlds.

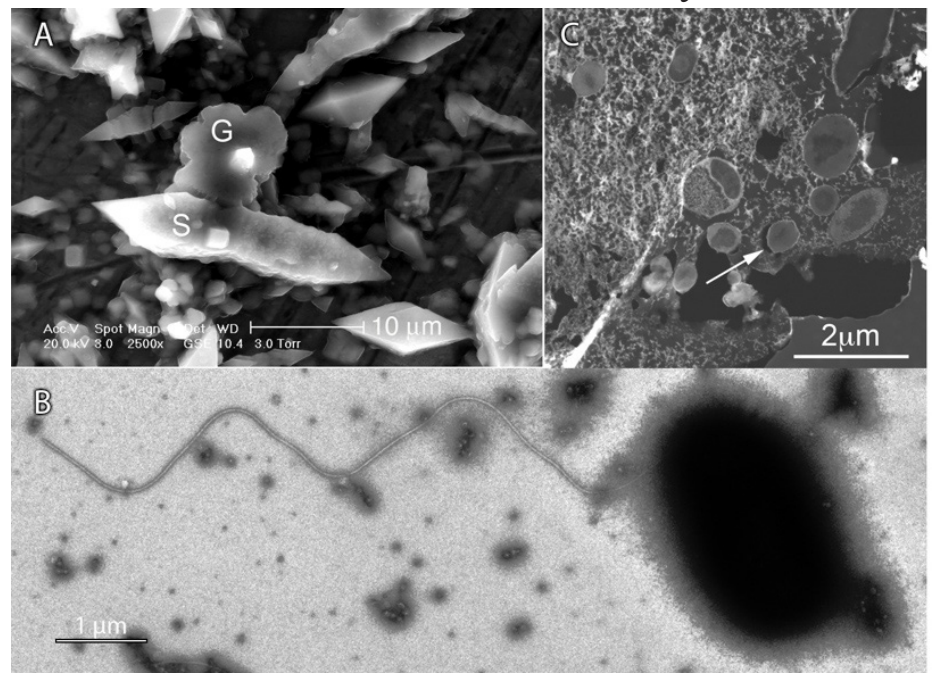

Fig. 3. Differerent EM techniques applied to analog samples. (A) ESEM image of a biofilm containing cells and microminerals. EDS identified the minerals as gypsum $(G)$ and sulfur $(S)$. Cells could not be definitively identified. (B) TEM image of stained but otherwise unprocessed marine bacterium. (C) STEM image of the same biofilm as in (A). This stained and thin-sectioned sample showed individual cells and "ghosts" of dead cells (arrow) surrounded by the mineral matrix.

Atomic Force. Atomic force microscopy (AFM) is part of the general class of scanning probe microscopy (SPM) which measures forces between a sharp tip and the sample surface. AFM is of increasing interest in microbiology ${ }^{26}$, as it is able to resolve filamentous structures and measure adhesive forces between organisms and substrates. It can also be coupled with fluorescence or used with functionalized tips to detect specific biochemistry. Its major drawback is very limited sample throughput capability requiring an additional survey instrument to preselect samples for high-resolution imaging.

\section{Technology Status and Development Recommendations}

Although microscopy is very well developed for laboratory biology, little has been done to apply it in field applications or planetary missions, particularly for astrobiology. Optical microimagers have flown on Mars missions ${ }^{27,28}$, but the highest resolution imager, at $6 \mu \mathrm{m}$, was still insufficient to detect bacteria (and it was never operated during spaceflight, being part of the failed Beagle 2 $\operatorname{lander}^{29}$ ). Two atomic-force microscopes (AFM) have been successfully flown ${ }^{30,31}$, but the limited survey capability made them useful for mineralogy only. A small number of microimagers targeted towards anticipated Ocean Worlds opportunities are currently in development: the ELVIS imaging system $^{32}, \mathrm{ELM}^{33}$, and ANTONIE ${ }^{34}$. The ELVIS imager is a combined sub-micron resolution DHM for high sample throughput label-free observations combined with a fluorescence imager to detect biotic chemistry on cell like objects. ELM is a bright field and fluorescence microscope with $<0.5$-micron resolution that uses both visible and deep-UV light to excite autofluorescence at four different wavelengths. ANTONIE is a combined optical imager for surveying samples and AFM 
for high resolution observation. All are intended for operation on liquid samples, and have begun work toward development of flyable instruments. Beyond these, there are a number of areas where development is needed to maximize our capability to eventually detect and characterize extraterrestrial life in situ.

All three of the systems in development above are designed for observing liquid samples to detect micron-scale objects. Observation of solid or granular samples, such as for many Mars environments, requires much more than simply removing the liquid sample holder and pointing the microscope at the target. Standard high-resolution compound objectives have depths of field of microns and are not suitable for observation of rough, unprepared samples. This can be addressed by developing sample processing systems that either collect and prepare suitable samples, prepare samples in situ, or with imaging systems that better accommodate the sample geometry. Sample processing systems will generally require designs specific to their target location and should be addressed by specific missions, but here we describe some optical systems that can perform well enough to detect bacteria on minimally processed samples.

DHM offers depths of field of $\sim 1 \mathrm{~mm}$ with $\sim 1 \mu \mathrm{m}$ resolution, and is available for laboratory and field use with minimally prepared samples. DHM in a reflected mode is more complex than working with dilute liquid samples because of the need to adjust relative intensities of object and reference beams, but this can be automated. Alternatively, FPM and related computational imaging techniques can achieve the desired resolution over millimeter depths, but at the expense of either large data volumes, or extensive on-board computing requirements.

For liquid environments where low concentrations of objects (biotic or abiotic) are expected, systems for concentration or sorting of samples will improve the chances of detecting any objects of interest. Filter concentration is used in laboratory environments, but the requirement to manage multiple filters for multiple samples add mass and system complexity. Optofluidic techniques, such as optical tweezers, microfluidic cell sorters, and fluidic or electro-fluidic traps can be used to trap and concentrate particles at bacterial scales, and in some cases can manipulate single objects to manage their observation in the microscope field or divert them to downstream instruments for sequential analysis of single small samples. These techniques are largely independent of the type of microscope used and exist currently at the laboratory benchtop level, but require development to be usable in planetary instruments.

Finally, the search for life in extreme environments necessitates searching through potentially large volumes of samples to find rare positive indicators. This leads to a data volume and processing issue for missions to outer planets and their moons, where available data transmission rates are low, round trip light-times are long, and energy may be limited. These problems are common to all instruments at these destinations, but we address specific needs for imaging systems here. Searching at micrometer pixel scale in volumes of many $\mathrm{cm}^{3}$ (or even 100s of $\mathrm{cm}^{3}$ ) results in a large volume of pixels with data that are not astrobiologically interesting, while at the same time we don't have a strong definition of what it means to detect life. To address this we recommend a combination of hardware and software development to support in situ data pre-processing of large volumes of data to prioritize the return of the data most likey to contain biomarkers or extant life indicators, which themselves need refinement.

Computing hardware available for space has been very limited in speed and storage. We recommend that NASA invest in expanding nascent programs to flight qualify, including modification if necessary, modern high performance computers for the environments expected at Europa and Enceladus. The Mars helicopter concept includes demonstration of Snapdragon processors in a 
relatively low radiation environment. Extending this development to include hardening for Europa and similar environments would be game-changing in the ability to evaluate data in situ, not just for microscopy, but for most other planetary instruments. On top of such modern processors, we recommend investment in programs to apply machine learning and related techniques to pre-processing of planetary instrument data. This should include algorithm development and training, with an emphasis on traceability to the physical phenomena that the algorithms are detecting, so that prioritization will be based on known physics, chemistry, and biology, even if we have an incomplete understanding of where complex abiotic chemistry ends and life begins.

\section{References}

1. Levin, G. V.; Straat, P. A. Nature 1979, 277, (5694), 326-326.

2. Klein, H. P. J Geophys Res-Planet 1998, 103, (E12), 28463-28466.

3. Neveu, M., et al. Astrobiology 2018, 18, (11), 1375-1402.

4. Hand, K. P., et al., Report of the Europa Science Definition Team, 2017.

5. Mckay, C. P. Space Sci Rev 2008, 135, (1-4), 49-54.

6. Heim, R., et al. Nature 1995, 373, (6516), 663-664.

7. Betzig, E., et al. Science 2006, 313, (5793), 1642-1645.

8. Hess, S. T., et al. Biophysical Journal 2006, 91, (11), 4258-4272.

9. Westphal, V., et al. Science 2008, 320, (5873), 246-249.

10. Lovelock, J. E. Nature 1965, 207, (4997), 568-\&.

11. Nadeau, J. L., et al. Adv Phys-X 2018, 3, (1), 221-246.

12. Lindensmith, C., et al. In 2018 IEEE Aerospace Conference, 3-10 March 2018; pp 1-9.

13. Bedrossian, M., et al. Astrobiology 2017, 17, (9), 913-925.

14. Sharma, S., et al. Nanotechnology 2018, 29, (13).

15. Marquet, P., et al. Optics Letters 2005, 30, (5), 468-470.

16. Zheng, G. A., et al. Nat Photonics 2013, 7, (9), 739-745.

17. Trask, B. J., et al. Cytometry 1982, 2, (4), 258-64.

18. Boss, E., et al. Opt Express 2018, 26, (9), 11125-11136.

19. Hairston PP, Ho J, Quant FR. J Aerosol Sci 1997, 28:471-82.

20. Hasegawa, N. Biocontrol Science 2013, 18, 4, 211-215.

21. Kang, S. et al., J. Biophonics 2020, 13,1, e201900190.

22. Schaefer, P. et al., Cytometry 2019, 95, 1, 34-46.

23. Gaskin, J. A., et al. In 2012 IEEE Aerospace Conference, 3-10 March 2012, 2012; pp 1-10.

24. Conley, J. M., et al. In Microbeam analysis - 1983. San Francisco, CA, San Francisco Press, Inc., pp 177-181.

25. Edmunson, J., et al. In 2018/03/1, p 2488.

26. Dufrene, Y. F. mBio 2014, 5, (4), e01363-14.

27. Herkenhoff, K. E., et al. J Geophys Res-Planet 2003, 108, (E12).

28. Edgett, K. S., et al. Space Sci Rev 2012, 170, (1-4), 259-317.

29. Thomas, N., et al. Planetary and Space Science 2004, 52, (9), 853-866.

30. Hecht, M. H., et al. J Geophys Res-Planet 2008, 113.

31. Bentley, M. S., et al. Acta Astronaut 2016, 125, 11-21.

32. Serabyn, E., et al.. In IEEE Aerospace Conference, IEEE: Big Sky Montana, 2019.

33. Chin, M., et al. In 42nd COSPAR Scientific Assembly, COSPAR: Pasadena, CA, 2018; p 2054.

34. Murray, A. E., et al. In Astrobiology Science Conference, Seattle, 2019; AGU: Seattle. 